\title{
Evaluation of Corneal Epithelial Healing Under Contact Lens with Spectral-Domain Anterior Segment Optical Coherence Tomography (SD- OCT)
}

\author{
Claudine E. Pang ${ }^{1}$, M. Vanathi ${ }^{2}$, Donald T.H. Tan ${ }^{1}$ and Jodhbir S. Mehta ${ }^{*}, 1$ \\ ${ }^{I}$ Singapore Eye Research Institute and Singapore National Eye Center, Singapore \\ ${ }^{2}$ Dr. Rajendra Prasad Centre for Ophthalmic Sciences, AIIMS, New Delhi, India
}

\begin{abstract}
Purpose: To describe a novel technique of using Spectral-domain (SD) anterior segment optical coherence tomography (SD-OCT) in the evaluation of corneal epithelial healing under a therapeutic contact lens (TCL) after lamellar keratoplasty and Epi-LASIK procedures.

Design: Prospective, non-comparative, observational case series.

Methods: Ten eyes of eight patients undergoing lamellar corneal transplantation and Epi-LASIK procedures at the Singapore National Eye Centre were included in the study. Ultra-high resolution SD-OCT scans of the cornea with a TCL in-situ were performed sequentially on the first, third and fifth day after procedure, with the RTVue (Optovue, Inc, Fremont, CA, USA), and the image findings were correlated with the clinical picture. Complete epithelial healing was verified with removal of TCL and fluorescein staining.

Results: 5 eyes underwent Descemet's stripping automated endothelial keratoplasty (DSAEK), 1 eye underwent deep anterior lamellar keratoplasty (DALK) and 4 eyes underwent Epi-LASIK. All eyes had complete epithelial healing with TCL in-situ by the third post-operative day. SD-OCT images were able to demonstrate the epithelial layer distinctly under the TCL in all cases.

Conclusions: SD-OCT is a valuable imaging tool for monitoring the progression of epithelial healing with TCL in situ in patients following corneal surgical procedures.
\end{abstract}

Keywords: Corneal epithelial healing, optical coherent tomography, contact lens, spectral domain.

\section{INTRODUCTION}

Anterior segment optical coherence tomography (ASOCT) is an increasingly important and fast evolving tool that obtains high-resolution, real-time, cross-sectional images of the cornea and anterior chamber [1-3]. Applications of ASOCT have been well described in the field of glaucoma/anterior segment surgery [4] with the evaluation of the drainage angle, trabeculectomy blebs and phakic intraocular lenses [5-8], as well as in corneal surgery where it is useful for the definition of both diagnostic $[9,10]$ and therapeutic $[1,11,12]$ procedures.

Conventional AS-OCT machines e.g. Visante (Carl Zeiss Meditec, Inc, Dublin, CA, USA) and slit-lamp OCT (Heidelberg, Heidelberg Engineering, Germany) use low coherence interferometry, with time-domain detection in which the echo delay of back-scattered light is measured by mechanically sweeping a mirror in a reference arm. This achieves imaging speeds of 400 axial scans per second [3, 13-15]. The development of a Spectral-domain detection system allows a dramatic improvement in the imaging speeds to 26,000 axial scans per second, resulting in ultra-

*Address correspondence to this author at the Cornea External Disease and Refractive Service, Singapore National Eye Centre, 11 Third Hospital Avenue, 168751, Singapore; Tel: + 65-62277255; Fax: + 65-62263395;

E-mail: jodmehta@gmail.com high resolution images which clearly distinguish the corneal sublayers including epithelium, Bowman's layer, Descemet's membrane and endothelium [2, 16-19].

Procedures such as lamellar corneal transplants and EpiLASIK (Laser-in-situ keratomileusis) involve the removal of corneal epithelium intra-operatively, and therapeutic contact lenses (TCL) are often used post-operatively to reduce pain and promote rapid re-epithelization. The timing of TCL removal is dependent upon assessment of complete epithelial healing, and TCLs should not be left in place longer than necessary, due to the potential for secondary infection, but it is often difficult to visualize the size of the remaining epithelial defect beneath the TCL without the use of fluorescein, which is generally contraindicated in the presence of a TCL due to lens staining. TCL removal is thus required to enable fluorescein staining to assess the epithelium, but carries the risk of traumatizing new epithelium which is easily detachable during the early phase of healing, and which may further delay full epithelization. With the increasing frequency of such procedures, the use of a non-invasive method for monitoring epithelium healing under the TCL will be advantageous so as to gauge the appropriate timepoint for TCL removal. The aim of this study is to describe the use of a commercially available Spectral-domain Anterior Segment Optical Coherence Tomography (SD-OCT) as an imaging tool to document the 
progression of epithelium healing under a TCL in postsurgical cases.

\section{METHODS}

This was a prospective, observational case series of patients from the Singapore National Eye Centre. A variety of cases were chosen to illustrate various situations with epithelial defects in which SD-OCT imaging may be useful. All patients had removal of epithelium during the primary surgical procedure: host epithelium in Descemet's stripping automated endothelial keratoplasty (DSAEK) and EpiLASIK; and donor epithelium in deep anterior lamellar keratoplasty (DALK). A TCL was applied post-operatively to facilitate epithelial healing as well as to improve postoperative patient comfort in DSAEK and Epi-LASIK cases $[20,21]$. In all cases, topical steroids and broad spectrum antibiotics were also prescribed.

The SD-OCT system RTVue (Optovue, Inc, Fremont, CA, USA) was used to image the cornea with TCL in-situ. Horizontal and vertical radial scans through the corneal vertex were carried out for each eye on central fixation, right gaze and left gaze to show sections across the central and peripheral cornea including the advancing edge of migrating epithelial tissue. Scans were taken at 1024 axial scans per line sampling with a scan acquisition time of 0.04 seconds per line. The Corneal Adaptor Module (CAM) high magnification lenses provided scan width up to $4 \mathrm{~mm}$ and transverse resolution of $10 \mu \mathrm{m}$.

The rate and nature of epithelial tissue migration was monitored with SD-OCT imaging taken on the first and third post-operative day in all patients until complete epithelial healing was noted. This was verified with the removal of the TCL and fluorescein application to confirm a fully epithelized, intact corneal surface.

\section{RESULTS}

10 eyes of 8 patients ( 4 male and 4 female, age ranging 21 to 77 years $($ mean $=46.25 \pm 19.5)$ were studied. Among these 5 eyes were right-sided and 5 were left-sided. 5 patients underwent Descemet's stripping automated endothelial keratoplasty (DSAEK) for Fuch's endothelial dystrophy [ $\mathrm{n}=3$ ] and pseudophakic bullous keratopathy $[\mathrm{n}=2], 1$ patient underwent deep anterior lamellar keratoplasty (DALK) for keratoconus and 2 patients underwent refractive correction by Epi-LASIK procedure.

In all eyes, the corneal epithelium was removed during the procedure (in the case of DALK, epithelium was removed from the donor cornea prior to graft placement) and a TCL was placed after the procedure. It was found that the SD-OCT images were able to show the corneal epithelium distinctly beneath the TCL including the leading edge of migrating epithelial tissue. Complete epithelial healing was noted on third post-operative day in all cases. Fluorescein staining after TCL removal confirmed the presence of a totally intact epithelium and correlated well with the SDOCT images (Fig. 1).

\section{DISCUSSION}

During the early post-operative course of keratoplasty and Epi-LASIK, re-epithelialization is critical for stabilization of visual acuity, graft transparency, graft survival, and protection of the stroma against melting [22]. Re-epithelialization is routinely enhanced with the use of a therapeutic contact lens (TCL) post-operatively [21, 23, 24]. This healing process is traditionally monitored clinically by direct visualization of the advancing edge on slit lamp biomicroscopy [25] or with the use of fluorescein staining [26]. The latter requires the removal of the TCL, which frequently results in discomfort to the patient and may also contribute to epithelial trauma which impedes the healing process of the epithelium. Removal and reinsertion of the TCL also increases the risk of infection in the immediate postoperative period. Hence, the benefits of using SD-OCT imaging as a non-invasive, non-contact method to monitor cornea re-epithelialization can easily be appreciated.

The newer SD-OCT technology eg. Spectralis (Heidelberg Engineering, Heidelberg, Germany) and Cirrus (Carl Zeiss Meditec, Jena, Germany) achieves higher speed and resolution $[2,16-19,27-32]$ than the conventional timedomain (TD) OCT technology and thus is able to provide exquisite details and delineation of fine corneal layers. In TD-OCT, the reference mirror moves one cycle for each axial scan, thus this mechanical movement limits the speed of image acquisition - resolution of the TD-OCT is inadequate to enable visualization of the corneal epithelium [2]. Furthermore, serial axial scanning is required as the detection system receives signal from a narrow range of depth at any one moment. In SD-OCT the reference mirror is kept stationary and spectral interferograms obtained between sample and reference reflections are transformed to provide axial scans. The absence of moving parts allows rapid acquisition of images and reflections from all layers in the sample are detected simultaneously $[18,19,30]$.

The RTVue has a speed of $26 \mathrm{kHz}$ and an axial resolution of $5 \mu \mathrm{m}$ (full-width-half-maximum) in tissue, which are respectively 13- and 3-fold improvements over a Visante TD-OCT (Carl Zeiss Meditec, Inc, Dublin, CA, USA) [6, 17, 18]. RTVue uses a shorter wavelength of $830 \mathrm{~nm}$ (compared to the Visante at $1310 \mathrm{~nm}$ ) and broader bandwidth, producing both better transverse and axial resolutions. With higher resolution and definition, smaller details in the cornea including the epithelial layer become visible.

The advantages of SD-OCT over TD-OCT are clearly higher speed and higher resolution, producing more detailed images. In addition, the high speed of SD-OCT allows measurement of corneal topography and corneal power. However, the spectral OCT is limited in its small scan range [31-35]. The RTVue is limited to approximately $6 \mathrm{~mm}$ scan width with the current adaptor lens, although this may be overcome with a different optical design. Due to this limited scan width, our study was unable to make accurate measurements of epithelial growth. A different adaptor lens that can image the entire cornea in one scan will be ideal to quantify the growth rate in future studies.

Another limitation is the strict trade-off between the scan depth and resolution because of the fixed pixel count of the line camera. The RTVue has a scan depth of only $2.3 \mathrm{~mm}$ in tissue, but may be extended by employing complex conjugate resolution. Due to the shorter $830 \mathrm{~nm}$-wavelength, the RTVue has poor ability to penetrate tissue that cause high amount of light scatter e.g. the sclera and limbus. This may pose as a problem in glaucoma imaging where angle 


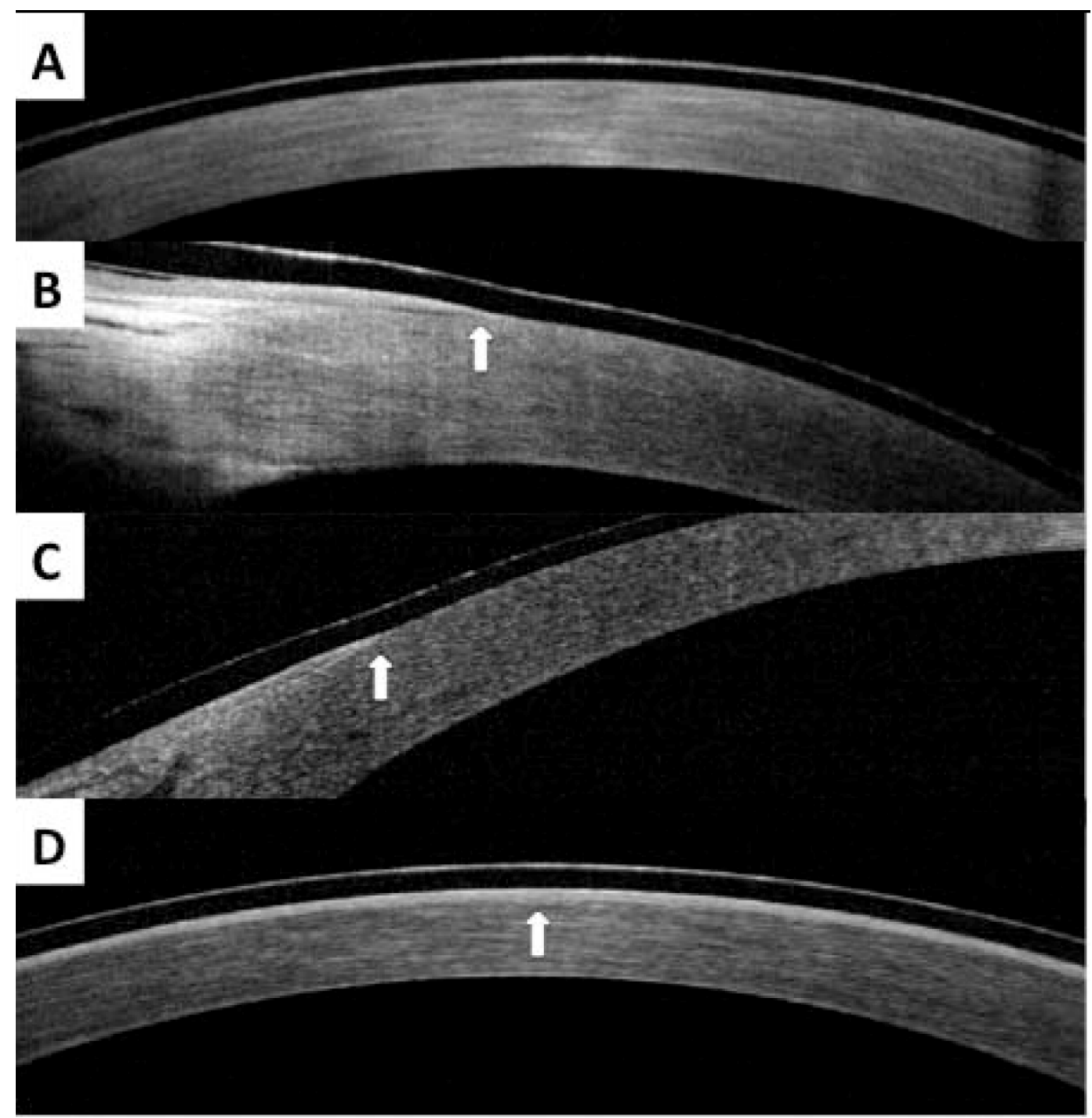

Fig. (1). SD-OCT images from patient in case report. A, First post-operative day over central cornea vertex, showing absence of epithelial layer under TCL. B, First post-operative day over nasal limbus, showing edge of epithelial layer (arrow). C, First post-operative day over temporal limbus, showing edge of epithelial layer (arrow). D, Third post-operative day, showing complete epithelial layer under TCL (arrow).

recess, iris root and ciliary body are poorly visible by blocking of the sclera. Wong et al. [36] showed that an adapted high definition OCT (modified Cirrus OCT mounted with a 60-diopter aspheric lens) was able to provide magnified views of the anterior chamber angle with visualization of Schwalbe's line and trabecular meshwork although not the ciliary body. Fortunately, the above limitations are less of a concern in cornea imaging.

Studies conducted to investigate the rate of normal epithelial healing have shown that for a $5 \mathrm{~mm}$ epithelial defect, complete wound closure occurs in $51 \pm 5.5 \mathrm{hrs}$, with a rate of $0.57 \pm 0.07 \mathrm{~mm}^{2} / \mathrm{hr}$ in the early phase of healing $(0$ $25 \mathrm{hr})$ and $0.21 \pm 0.06 \mathrm{~mm}^{2} / \mathrm{hr}$ in the later phase $(30-55 \mathrm{hr})$ [37, 38]. Our study was consistent with the reported rates as we were able to show that all cases had complete epithelial healing by the third day post procedure. Future studies where direct measurement of the epithelial defect can be done to calculate the exact rate of wound closure.
As shown in our study, SD-OCT images are able to demonstrate the epithelial layer under the TCL very distinctly. Hence, SD-OCT is an excellent tool for monitoring re-epithelialization. It negates the need of TCL removal or fluorescein application during the healing phase and avoids any unnecessary micro-trauma to the epithelium, patient discomfort or risk of infection during the process. Direct slit lamp biomicroscopic visualization of the healing epithelium can be difficult in cases of edematous corneas following DSAEK especially with a TCL in-situ. The use of SD-OCT can be applied to a number of corneal conditions apart from post-operative epithelial healing including monitoring the progression and epithelial healing response of ocular surface disorders including neurotrophic keratitis, recurrent corneal erosions, metaherpetic corneal ulceration and other forms of delayed epithelial healing to treatment, documenting the depth of lesions in stromal dystrophies, and assessing patients' post-refractive corneal surgical procedures. 
The main drawback of this study is the limited number of study subjects, and this is primarily because the usage of SDOCT in this study is unprecendented and this study serves as a pilot for future studies. We also acknowledge the fact that the state-of-the-art SD-OCT may not be readily available in all clinical settings and hence future studies are necessary to investigate the cost effectiveness of the SD-OCT for routine use.

In summary, SD-OCT demonstrates the ability to monitor the corneal epithelial growth under a TCL in postsurgical cases with high-resolution images and will likely be a useful tool in the diagnostic management of corneal patients.

\section{SUPPORT}

Grant from Singapore National Eye Centre, Singapore.

\section{REFERENCES}

[1] Lim LS, Aung HT, Tin A, et al. Corneal imaging with anterior segment optical coherence tomography for lamellar keratoplasty procedures. Am J Ophthalmol 2008;145: 81-90.

[2] Potsaid B, Gorczynska I, Srinivasan VJ, et al. Ultrahigh speed spectral / Fourier domain OCT ophthalmic imaging at 70,000 to 312,500 axial scans per second. Opt Exp 2008; 6: 15149-69.

[3] Huang D, Swanson EA, Lin CP. Optical coherence tomography. Science $1991 ; 254: 1179-81$.

[4] Radhakrishnan S, Rollins AM, Roth JE, et al. Real-time optical coherence of the anterior segment at $1310 \mathrm{~nm}$. Arch Ophthalmol 2001;119:1179-85.

[5] Wang BS, Wang NL. Applications of anterior segment optical coherence tomography in ophthalmology. Zhonghua Yan Ke Za Zhi 2008; 44:185-8.

[6] Ramos JL, Li Y, Huang D. Clinical and research applications of anterior segment optical coherence tomography - a review. Clin Experiment Ophthalmol 2009; 37(1): 81-9.

[7] Simpson T, Fonn D. Optical coherence tomography of the anterior segment. Ocul Surf 2008; 6: 117-27.

[8] Konstantopoulos A, Hossain P, Anderson DF. Recent advances in ophthalmic anterior segment imaging: a new era for ophthalmic diagnosis? Br J Ophthamol 2007; 91: 551-7.

[9] Haque S, Simpson T, Jones L. Corneal and epithelial thickness in keratoconus: a comparison of ultrasonic pachymetry, Orbscan II, and optical coherence tomography. J Refract Surg 2006; 22: 48693.

[10] Wirbelauer C, Pham DT. Imaging and quantification of calcified corneal lesions with optical coherence tomography. Cornea 2004; 23: 439-42.

[11] Wang J, Thomas J, Cox I, et al. Non-contact measurements of central corneal epithelial and flap thickness after laser in situ keratomileusis. Invest Ophthalmol Vis Sci 2004; 45:1812-16.

[12] Maldonado MJ, Ruiz-Oblitas L, Munuera JM, et al. Optical coherence tomography evaluation of the corneal cap and stromal bed features after laser in situ keratomileusis for high myopia and astigmatism. Ophthalmology 2000; 107: 81-7.

[13] Hitzenberger CK. Optical measurement of the axial eye length by laser Doppler interferometry. Invest Ophthalmol Vis Sci 1991; 32: 616-24.

[14] Swanson EA, Izatt JA, Hee MR, et al. In vivo retinal imaging by optical coherence tomography. Opt Lett 1993; 18: 1864-66.

[15] Haberland U, Jansen P, Blazek V, Schmitt HJ. Optical coherence tomography of scattering media using frequency-modulated continuous-wave techniques with tunable near-infrared laser. Coherence Domain Opt Methods Biomed Sci Clin Appl 1997:2981:20-28.
[16] Chen TC, Cense B, Pierce MC, et al. Spectral domain optical coherence tomography: ultra-high speed, ultra-high resolution ophthalmic imaging. Arch Ophthalmol 2005; 123: 1715-20.

[17] Wojtkowski M, Bajraszewski T, Targowski $\mathrm{P}$, et al. Real-time in vivo imaging by high-speed spectral optical coherence tomography. Opt Lett 2003; 28: 1745-7.

[18] Choma M, Sarunic M, Yang C, et al. Sensitivity advantage of swept source and Fourier domain optical coherence tomography. Opt Exp 2003; 11: 2183-9.

[19] de Boer JF, Cense B, Park BH, et al. Improved signal-to-noise ratio in spectral-domain compared with time-domain optical coherence tomography. Opt Lett 2003; 28: 1067-9.

[20] Palmer RM, McDonald MB. A corneal lens/shield system to promote postoperative corneal epithelial healing. J Cat Ref Surg 1995; $21: 125-6$.

[21] Lim L, Tan DT, Chan WK. Therapeutic use of Bausch \& Lomb PureVision contact lenses. CLAO J 2001; 27: 179-85.

[22] Sinha R, Tandon R, Titliyal JS. Unilateral versus bilateral patching: Management of a post-keratoplasty epithelial defect in a case of congenital hereditary endothelial dystrophy with manifest latent nystagmus. J Ped Ophthalmol Strab 2003; 40: 206-8.

[23] Arora R, Jain S, Monga S, et al. Efficacy on continuous wear PureVision contact lenses for therapeutic use. Cont Lens Anterior Eye 2004; 27: 39-43.

[24] Montero J, SparholtJ, Mely R. Retrospective case series of therapeutic applications of a Iotrafilcon A silicone hydrogel soft contact lens. Eye Contact Lens 2003; 29: S54-9.

[25] Evans MDM, McFarland GA, Taylor S, et al. The response of healing epithelium to grooved polymer surfaces. Biomaterials 2005; 26: 1703-11.

[26] Dean SJ, Novitskaya ES, Moore TCB, et al. Documentation of corneal epithelial defects with fluorescein-enhanced digital fundus camera photography. Clin Exp Ophthalmol 2008; 36:113-8.

[27] Kaluzny BJ, Kaluzny JJ, Szkulmowska A, et al. Spectral optical coherence tomography: a novel technique for cornea imaging. Cornea 2006; 25: 960-5.

[28] Wojtkowski M, Kowakzyk A, Targowski P, et al. Frequency domain optical coherence tomography techniques in eye imaging. Acta Physica Polonica A 2002; 102: 739-46.

[29] Fercher AF, Hitzenberger CK, Kamp G, et al. Measurement of intraocular distances by backscattering spectral interferometry. Opt Commun 1995; 117: 43-8.

[30] Leitgeb R, Hitzenberger CK, Fercher AF. Performance of fourierdomain $v s$ time domain optical coherence tomography. Opt Exp 2003; 11: 889-94.

[31] Nassif N, Cense B, Park BH, et al. In vivo human retinal imaging by ultrahigh-speed spectral domain optical coherence tomography. Opt Lett 2004; 29: 480-2.

[32] Wojtkowski M, Kowakzyk A, Leitgeb R, et al. Full range complex spectral optical coherence tomography technique in eye imaging. Opt Lett 2002; 27: 1415-17.

[33] Nassif NA, Cense B, Park BH, et al. In vivo high resolution videorate spectral domain optical coherence tomography of the human retina and optic nerve. Opt Exp 2004; 12: 367-76.

[34] Yun SH, Tearney GJ, Bourma BE, et al. High-speed spectral domain optical coherence tomography at $1.3 \mathrm{um}$ wavelength. Opt Exp 2003; 11: 3598-604.

[35] Bower B, Izatt JA. Rapid volumetric imaging of the human retina in vivo using a low-cost, spectral domain optical coherence tomography system. Invest Ophthalmol Vis Sci 2005; 46: 1050.

[36] Wong HT, Lim MC, Sakata LM, et al. High-definition optical coherence tomography imaging of the iridocorneal angle of the eye. Arch Ophthalmol 2009; 127: 256-60.

[37] Tanelian DL, Bisla K. A new in vitro corneal preparation to study epithelial wound healing. Inves Ophthalmol Vis Sci 1992; 33: 3024-38.

[38] Wilson SA, Last A, Management of corneal abrasions. Am Fam Physician 2004; 70: 123-30. 